\title{
Endoscopic Procedure
}

National Cancer Institute

\section{Source}

National Cancer Institute. Endoscopic Procedure. NCI Thesaurus. Code C16546.

A diagnostic or therapeutic procedure in which an endoscope is inserted in a tubular organ to examine the structural architecture and/or remove abnormal tissues. 